Astrophysics and Space Science manuscript No.

(will be inserted by the editor)

S. Dall'Osso • L. Stella

\title{
Newborn Magnetars as sources of Gravitational Radiation: constraints from High Energy observations of Magnetar Candidates
}

Received: date / Accepted: date

\begin{abstract}
Two classes of high-energy sources, the Soft Gamma Repeaters and the Anomalous X-ray Pulsars are believed to contain slowly spinning "magnetars", i.e. neutron stars the emission of which derives from the release of energy from their extremely strong magnetic fields $\left(>10^{15} \mathrm{G}\right)$. The enormous energy liberated in the 2004 December 27 giant flare from SGR 1806-20 $\left(\sim 5 \times 10^{46} \mathrm{erg}\right)$, together with the likely recurrence time of such events, points to an internal magnetic field strength of $\geq 10^{16} \mathrm{G}$. Such strong fields are expected to be generated by a coherent $\alpha-\Omega$ dynamo in the early seconds after the Neutron Star (NS) formation, if its spin period is of a few milliseconds at most. A sub'stantial deformation of the NS is caused by such fields and, provided the deformation axis is offset from the spin axis, a newborn millisecond-spinning magnetar would thus radiate for a few days a strong gravitational wave signal the frequency of which $(\sim 0.5-2 \mathrm{kHz}$ range) decreases in time. This signal could be detected with Advanced LIGO-class detectors up to the distance of the Virgo cluster, where $\geq 1 \mathrm{yr}^{-1}$ magnetars are expected to form. Recent X-ray observations revealed that SNRs around magnetar candidates do not appear to have received a larger energy input than in standard SNRs (Vink \& Kuiper 2006). This is at variance with what would be expected if the spin energy of the young, millisecond NS were radiated away as electromagnetic radiation andd/or relativistic particle winds. In fact, such energy would be transferred quickly and efficiently to the expanding gas shell. This may thus suggest that magnetars did not form with the expected very fast initial spin. We show here that these findings can be reconciled
\end{abstract}

\section{S. Dall'Osso}

INAF-Osservatorio Astronomico di Roma

via di Frascati 33, 00040, Monteporzio Catone (Roma)

Tel.: +390694286437 E-mail: dallosso@mporzio.astro.it

L. Stella

INAF-Osservatorio Astronomico di Roma

via di Frascati 33, 00040 Monteporzio Catone (Roma)

Tel.: +390694286436 E-mail: stella@mporzio.astro.it with the idea of magnetars being formed with fast spins, if most of their initial spin energy is radiated thorugh GWs. In particular, we find that this occurs for essentially the same parameter range that would make such objects detectable by Advanced LIGO-class detectors up to the Virgo Cluster. If our argument holds for at least a fraction of newly formed magnetars, then these objects constitute a promising new class of gravitational wave emitters.

Keywords gravitational waves - stars: magnetic fields — stars: neutron — stars: individual: SGR 1806-20)

PACS 97.60.Jd $\cdot 97.60 . \mathrm{Bw} \cdot 04.30 . \mathrm{Db} \cdot 95.85 . \mathrm{Sz}$

\section{Introduction}

The Soft Gamma Repeaters, SGRs, and the Anomalous $\mathrm{X}$-ray Pulsars, AXPs, have a number of properties in common (Mereghetti \& Stella 1995; Kouveliotou et al. 1998; Woods \& Thompson 2004). They have spin periods of $\sim 5 \div 10 \mathrm{~s}$, spin-down secularly with $\sim 10^{4} \div 10^{5} \mathrm{yr}$ timescale, are isolated and in some cases associated to supernova remnants with $\sim 10^{3} \div 10^{4}$ yr ages. Rotational energy losses are $10 \div 100$ times too low to explain the $\sim 10^{34} \div 10^{35} \mathrm{erg} / \mathrm{s}$ persistent emission of these sources. Both AXPs and SGRs have periods of intense activity during which recurrent, subsecond-long bursts are emitted (peak luminosities of $\sim 10^{38} \div 10^{41} \mathrm{erg} / \mathrm{s}$ ). The initial spikes of giant flares have comparable duration but 3 to 6 orders of magnitude larger luminosity. Giant flares are rare, only three have been observed in about $30 \mathrm{yr}$ of monitoring. Given the highly super-Eddington luminosities of recurrent bursts and, especially, giant flares, accretion models are not viable.

In the magnetar model, SGRs and AXPs derive their emission from the release of the energy stored in their extremely high magnetic fields (Duncan \& Thompson 1992; Thompson \& Duncan 1993, 1995, 1996, 2001). This is the leading model for interpreting the unique features of these sources. According to it, a wound-up, mainly 
toroidal magnetic field characterizes the neutron star interior $\left(B>10^{15} \mathrm{G}\right)$.

The emerged (mainly poloidal) field makes up the neutron star magnetosphere; dipole strenghts $\left(B_{d} \sim\right.$ few $\times 10^{14} \mathrm{G}$ ) are required to generate the observed spindown (Thompson \& Duncan 1993; Thompson \& Murrav 2001). Impulsive energy is fed to the neutron star magnetosphere through Alfvén waves driven by local "crustquak and producing recurrent bursts with a large range of amplitudes. Giant flares likely originate in large-scale rearrangements of the toroidal inner field or catastrophic instabilities in the magnetosphere (Thompson \& Duncan 2001; Lyutikov 2003). Most of this energy breaks out of the magnetosphere in a fireball of plasma expanding at relativistic speeds which produces the initial spike of giant flares. The oscillating tail that follows this spike, displaying many tens of cycles at the neutron star spin, is interpreted as due to a "trapped fireball", which remains anchored inside the magnetosphere (the total energy released in this tail is $\sim 10^{44} \mathrm{erg}$ in all three events detected so far, comparable to the energy of $\mathrm{a} \sim 10^{14} \mathrm{G}$ trapping magnetospheric field).

\section{The 2004 December 27 Event and the Internal Magnetic field of Magnetars}

The 2004 December 27 giant flare from SGR1806-20 provides a new estimate of the internal field of magnetars. About $5 \times 10^{46}$ erg were released during the $\sim 0.6 \mathrm{~s}$ long initial spike of this event (Terasawa et al. 2005; Hurley et 2005). This is more than two decades higher than the energy of the other giant flares observed so far, the 1979 March 5 event from SGR 0526-66 (Mazets et al. 1979) and the 1998 August 27 event from SGR 1900+14 (Hurley 1999; Feroci et al. 1999). Only one such powerful flare has been recorded in about $30 \mathrm{yr}$ of monitoring of the $\sim 5$ known magnetars in SGRs. The recurrence time in a single magnetar implied by this event is thus about $\sim$ $150 \mathrm{yr}$. The realisation that powerful giant flares could be observed from distances of tens of Mpc (and thus might represent a sizeable fraction of the short Gamma Ray Burst population) motivated searches for 2004 Dec 27like events in the BATSE GRB database Lazzati et al. 2005; Popov \& Stern 2005). The upper limits on the recurrence time of powerful giant flares obtained in these studies range from $\tau \sim 130$ to 600 yr per galaxy, i.e. $\sim 4$ to 20 times longer than inferred above. Therefore these authors conclude that on 2004 Dec 27 we have witnessed a "statistically unlikely" event. On the other hand Tanvir et al. (2005) find that the location of 10$25 \%$ of the short GRBs in the BATSE catalogue correlates with the position of galaxies in the local universe $(<110 \mathrm{Mpc})$, suggesting that a fraction of the short GRBs may originate from a population of powerful Giant Flare-like events.
A 2004 Dec 27-like event in the Galaxy could not be missed, whereas several systematic effects can reduce the chances of detection from large distances (see e.g. the discussion in Lazzati et al. (2005); Nakar et al. (2005)). Rather than regarding the 2004 Dec 27 event as statistically unlikely, one can thus evaluate the chances of a recurrence time of hundreds of years, given the occurrence of the 2004 Dec 27 hyperflare. We estimate that, having observed a powerful giant flare in our galaxy in $\sim 30$ yr of observations, the Bayesian probability that the galactic recurrence time is $\tau>600 \mathrm{yr}$ is $\sim 10^{-3}$, whereas the $90 \%$ confidence upper limit is $\tau \sim 60 \mathrm{yr}$. We thus favor smaller values and assume in the following $\tau \sim 30 \mathrm{yr}$.

In $\sim 10^{4}$ yr (that we adopt for the SGR lifetime), about 70 very powerful giant flares should be emitted by an SGR, releasing a total energy of $\sim 4 \times 10^{48} \mathrm{erg}$. We note that if the giant flares' emission were beamed in a fraction $b$ of the sky (and thus the energy released in individual flares a factor of $b$ lower), the recurrence time would be a factor of $b$ shorter. Therefore the total release of energy would remain the same. If this energy originates from the magnetar's internal magnetic field, this must be $\geq 10^{15.7} \mathrm{G}$ (Stella et al. 2005; Terasawa et al. 2005). This value should be regarded as a lower limit. Firstly, the magnetar model predicts a conspicuous neutrino luminosity from ambipolar diffusion-driven field decay, an energy component that is not available to flares. Including this, we estimate that the limit above increases by $\sim 60 \%$ and becomes $B \geq 10^{15.9} \mathrm{G}$. Secondly, ambipolar diffusion and magnetic dissipation should take place at a faster rate for higher values of the field (Thompson \& Duncan 1996). Therefore estimates of the internal B-field based on present day properties of SGRs likely underestimate the value of their initial magnetic field.

Very strong toroidal B fields are expected to be generated inside a differentially rotating fast spinning neutron star, subject to vigorous neutrino-driven convection instants after its formation (Duncan \& Thompson 1992). A field of several $\times 10^{16} \mathrm{G}$ can be generated in magnetars that are born with spin periods of a few milliseconds (Thompson \& Duncan 1993). As discussed by Duncan (1998), values up to $\sim 10^{17} \mathrm{G}$ cannot be ruled out.

In the following we explore the consequences of these fields for the generation of gravitational waves from newborn magnetars. We parametrize their (internal) toroidal field with $B_{t, 16.3}=B_{t} / 2 \times 10^{16} \mathrm{G}$, (external) dipole field with $B_{d, 14}=B_{d} / 10^{14} \mathrm{G}$ and initial spin period with $P_{i, 2}=P_{i} /(2 \mathrm{~ms})$.

\section{Magnetically-Induced Distortion and Gravitational Wave Emission}

The possibility that fast-rotating, magnetically-distorted neutron stars are conspicuous sources of gravitational radiation has been discussed by several authors (Bonazzola \& Marck 
1994; Bonazzola \& Gourgoulhon 1996). More recently, work has been carried out in the context of the magnetar model, for internal magnetic fields strengths of $\sim 10^{14}-10^{16} \mathrm{G}$ (Konno. Obata. Koiima 2000; Palomba 2001; Cutler 2002). In the following we show that for the range of magnetic fields discussed in Section 2, newly born, millisecond spinning magnetars are conspicuous sources of gravitational radiation that will be detectable up to Virgo cluster distances (Stella et al. 2005).

The anisotropic pressure from the toroidal B-field deforms a magnetar into a prolate shape, with ellipticity $\epsilon_{B} \sim-6.4 \times 10^{-4}\left(<B_{t, 16.3}^{2}>\right)$, where the brackets indicate a volume-average over the entire core Cutler 2002). As long as the axis of the magnetic distortion is not aligned with the spin axis, the star's rotation will cause a periodic variation of the mass quadrupole moment, in turn resulting in the emission of gravitational waves, GWs, at twice the spin frequency of the star.

Free precession of the ellipsoidal NS is also excited and, as shown by Mestel \& Takhar (1972) and Cutler (2002), its viscous damping drives the symmetry axis of the magnetic distortion orthogonal to the spins axis, if the ellipsoid is prolate, i.e. if the magnetic field is toroidal. Therefore, viscous damping of free precession in newly born magnetars leads to a geometry that maximizes the time-varying mass quadrupole moment, and GW emission accordingly.

However, the power emitted in GWs scales as $\propto P^{-6}$. Therefore the GW signal, for a given toroidal B-field, depends critically on the initial value and early evolution of the spin period. The spin evolution of a newborn magnetar is determined by angular momentum losses from GWs, electromagnetic dipole radiation and relativistic winds. According to Thompson et al (2004), the latter mechanism is negligible except for external dipole fields $<(6 \div 7) \times 10^{14} \mathrm{G}$ and we will neglect it here.

The spin evolution of a newborn magnetar under the combined effects of GW and electromagnetic dipole radiation is given by

$\dot{\omega}=-K_{d} \omega^{3}-K_{\mathrm{gw}} \omega^{5}$,

where $\omega=2 \pi / P$ is the angular velocity,

$K_{d}=\left(B_{d}^{2} R^{6}\right) /\left(6 I c^{3}\right)$ and $K_{\mathrm{gw}}=(32 / 5)\left(G / c^{5}\right) I \epsilon_{B}^{2}$, with $R$ the neutron star radius, $G$ the gravitational constant and $c$ the speed of light. This gives a spin-down timescale of

$\tau_{s d} \equiv \frac{\omega}{2 \dot{\omega}} \simeq 10 P_{i, 2}^{2}\left(B_{d, 14}^{2}+1.15 B_{t, 16.3}^{4} P_{i, 2}^{-2}\right)^{-1} \mathrm{~d}$

The condition for the newly formed magnetar to become an orthogonal rotator before loosing a significant fraction of its initial spin energy is (Stella et al. 2005):

$\frac{\tau_{s d}}{\tau_{\text {ort }}} \simeq 26 \frac{B_{t, 16.3}^{2}}{B_{d, 14}^{2} P_{i, 2}^{-1}+1.15 B_{t, 16.3}^{4} P_{i, 2}^{-3}}>1$.

If condition (3) is met, the magnetar quickly becomes a maximally efficient GW emitter, while its spin period is still close to the initial one. In this case, the instantaneous signal strain can be expressed as:

$h \sim 3 \times 10^{-26} d_{20}^{-1} P_{2}^{-2} B_{t, 16.3}^{2}$,

where the distance $d_{20}=d /(20 \mathrm{Mpc})$ is in units of the Virgo Cluster distance and the angle-averaged strain is that given by Ushomirsky et al (2000). We estimate the characteristic amplitude, $h_{c}=h N^{1 / 2}$, where $N \simeq \tau_{s d} / P_{i}$ is the number of cycles over which the signal is observed. Using equation (4) we obtain:

$$
h_{c} \simeq 6 \times 10^{-22} \frac{B_{t, 16.3}^{2}}{d_{20} P_{i, 2}^{3 / 2}\left(B_{d, 14}^{2}+1.15 B_{t, 16.3}^{4} P_{i, 2}^{-2}\right)^{\frac{1}{2}}}
$$

Under the conditions discussed above, strong GW losses are not quenched immediately after the magnetar birth but rather extend in time, typically from days to a few weeks, before fading away as a result of the star spindown. The characteristic amplitude in eq. (5) is within reach of GW interferometers of the Advanced LIGO class. In order to assess the detectability of these GW signal we compute the optimal (matched-filter) signal-to-noise ratio for a signal sweeping the $500 \mathrm{~Hz}-2 \mathrm{kHz}$ band by using the current baseline performance of Advanced LIGO (details are given in Stella et al. (2005)). Fig. 1 shows lines of constant $S / N$ for a source at $d_{20}=1$ and selected values of the initial rotation period $\left(P_{i}=1.2,2\right.$ and $2.5 \mathrm{~ms})$ in the $\left(B_{t}, B_{d}\right)$ plane: $\mathrm{GWs}$ from newborn magnetars can produce $S / N>8$ for $B_{t} \geq 10^{16.5} \mathrm{G}$ and $B_{d} \leq 10^{14.5} \mathrm{G}$. This is the region of parameter space that offers the best prospects for detection as we now discuss. Matched-filtering represents the optimal detection strategy for long-lived, periodic signals, also in those cases where their frequency is slowly evolving over time. This process involves the correlation of the data stream with a discrete set of template signals that probe the relevant space of unknown parameters: the sky position (2 extrinsic parameters) and the 2 intrinsic parameters that control the evolution of the $\mathrm{GW}$ phase (see Eq.(10). Template spacing in the parameter space is chosen appropriately, so as to reduce to a value less than, say, $10 \%$ (depending on the sensitivity one wants to achieve) the fraction of the intrinsic signal-to-noise ratio that is lost in the cross-correlation. Dall'Osso \& Re (2006) have recently investigated in detail a matchedfiltering (coherent) search strategy for the expected signal from newly formed magnetars in the Virgo cluster. Given the two (uncorrelated) parameters involved in the signal frequency evolution, this approach implies an unaffordable computational cost. A huge number of templates $\sim 10^{18}$ would be required to cover the relevant parameter space (the plane $B_{d}$ vs. $B_{t}$ ) with a sufficiently fine grid that the intrinsic signal-to-noise ratio would not be too degraded. Although this calculation is rather idealized, the resulting number of templates is so large that no realistic calculation could decrease it by the several orders of magnitude needed to make the search feasible. The search for this new class of signals represents 


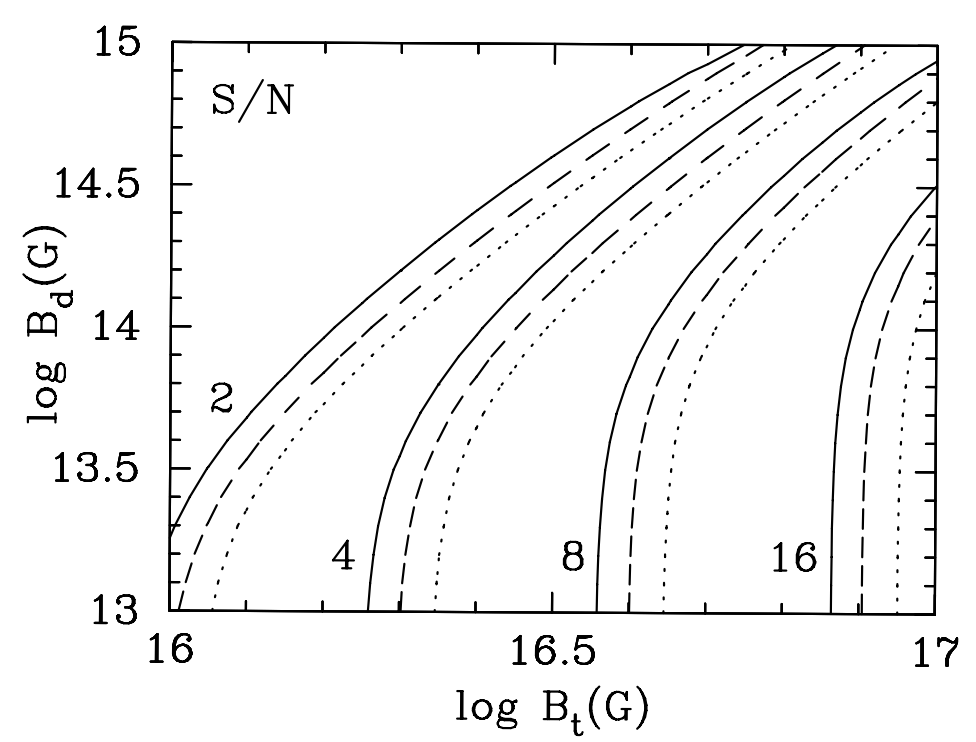

Fig. 1 Lines of constant $S / N$ for selected values of the initial spin period in the internal toroidal magnetic field, $B_{t}$, and external dipole field, $B_{t}$, plane for a source at the distance of the Virgo Cluster $\left(d_{20}=1\right)$. Solid, dashed and dotted curves correspond to an initial spin period of $P_{i}=1.2,2$ and $2.5 \mathrm{~ms}$, respectively. The calculations take into account the time required for the toroidal magnetic field axis to become orthogonal to the spin axis. Note that according to Thompson et al (2004), strong angular momentum losses by relativistic winds set in and dominate the spin down for $B_{d}>6 \div 7 \times 10^{14} \mathrm{G}$; the curves for such values of $B_{d}$ should thus be treated with caution.

however a challenge that must be investigated in greater depth. We are currently studying a hierarchical approach to the problem, a process where coherent and incoherent stages of the search are alternated as to reduce the computational requirements by a large factor, at the price of a relativeley modest loss in sensitivity to the signal.

\section{Observational Constraints on GW emission from newly formed magnetars}

A NS spinning at $\sim$ ms period has a spin energy $E_{\text {spin }} \approx$ $2.8 \times 10^{52}\left(P_{i} / 1 \mathrm{~ms}\right)^{-2}$ erg and the spindown timescale through magnetic dipole radiation and/or relativistic particle winds is extremely short, from a few weeks to $\sim$ one day for dipole fields in the $\left(10^{14} \div 10^{15}\right) \mathrm{G}$ range. In standard magnetar scenarios, most of the initial spin energy is expected to be rapidly transferred to the surrounding supernova ejecta through these spindown mechanisms (Thompson et al 2004). Therefore, present-day SNRs aroun known magnetar candidates should bear the signature of such a large energy injection. For initial spin periods less than $3 \mathrm{~ms}$, the injected energy would be $>3.5 \times 10^{51}$ erg, making these remnants significantly more energetic than those surrounding ordinary NSs $\left(\leq 10^{51} \mathrm{erg} \mathrm{s}^{-1}\right)$. The X-ray spectra of the SNRs surrounding known magnetar candidates (two APXs and two SGRs) studied by Vink et al. (2006), do not show any evidence that their total energy content differs from that in remnants surrounding common NSs $\left(\approx 10^{51} \mathrm{erg}\right)$ : this result constrains magnetar parameters at birth. Vink \& Kuiper
(2006) deduced from their measurements an initial spin $P_{i} \geq(5 \div 6) \mathrm{ms}$ for the above mentioned sources, assuming that all the spin-down energy is emitted through electromagnetic radiation and/or particle winds, and thus absorbed by the surrounding ejecta in the early days of spindown. Their limit period is long enough to rise a serious question as to the viability of the $\alpha-\Omega$ dynamo scenario for generating the large-scale magnetic fields of magnetars. Models that do not rely upon very short spin periods at birth, such as the flux-freezing scenario suggested by Ferrario \& Wickramasinghe (2006), would be favored by these results.

However, given the possibility that newly formed magnetars be strong GW emitters in their early days, we show that the results by Vink \& Kuiper (2006) can be accounted for within this framework. Most of a magnetar's initial spin energy could indeed be released through GWs, without being absorbed by the expanding remnant shell. Therefore, the results of X-ray studies can be used within our model to constrain the initial combination of $\mathbb{P}_{i}, B_{d}, B_{t}$.

\subsection{Strong GW emission at birth?}

The general expression for the total energy emitted via GWs is given by:

$E_{\mathrm{gw}}^{\mathrm{TOT}}=-\int_{t_{i}}^{\infty} \dot{E}_{\mathrm{gw}} d t=-\int_{\omega_{i}}^{0} \frac{\dot{E}_{\mathrm{gw}}}{\dot{\omega}} d \omega$ 


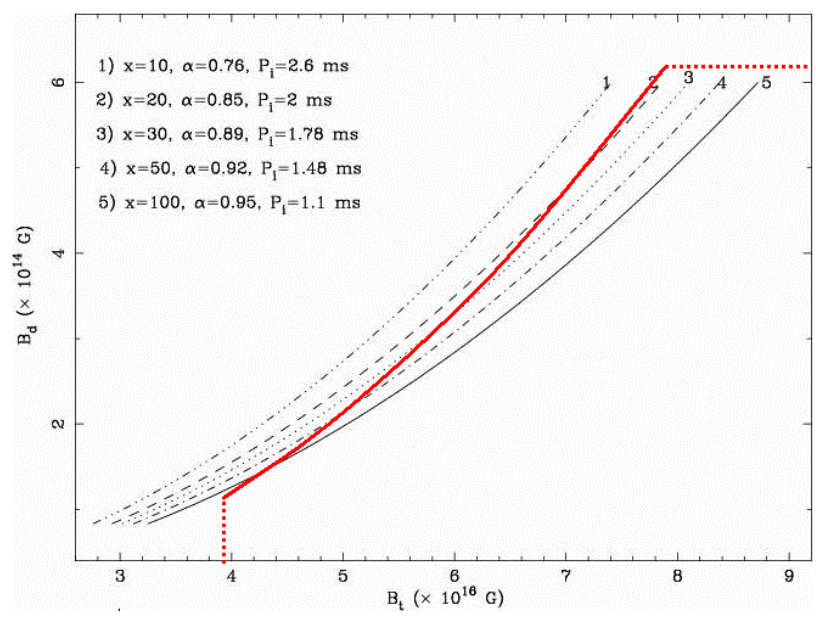

Fig. 2 Loci in the $B_{d}$ vs. $B_{t}$ plane for which $E_{S N}^{\mathrm{inj}} \simeq 10^{51}$ ergs. Curves are labelled for different values of the ratio $(x)$ of the GW to magnetodipole torques. Through Eq. (10), it is seen that fixing this ratio is equivalent to defining the corresponding initial spin. The corresponding locus is thus uniquely determined through the first expression in Eq. (9). Note that, given $x$, the corresponding curve shifts to the right for decreasing values of $E_{S N R}^{\text {inj }}$. The red curve represents the $\mathrm{S} / \mathrm{N}=8$ curve of Fig. (1). In the framework of our model, thus, fast spinning newly formed magnetars that do emit $\leq 10^{51}$ ergs of spin-down energy through electromagnetic radiation can be expected to be detectable with LIGO II up to the distance of the Virgo cluster. Stated the other way, objects whose GW signal could be detectable by LIGO II up to the distance of the Virgo Cluster would inject - as found in the case of magnetar candidates in the Galaxy - $\leq 10^{51}$ ergs in the expanding shells of the surrounding SNR.

We insert Eq. (1) and the expression for the GW luminosity of an elliptically distorted, spinning object into Eq. (6) to obtain the following analytical solution:

$E_{\mathrm{gw}}^{\mathrm{TOT}}=I \int_{0}^{\omega_{i}} \frac{\omega^{3}}{\omega^{2}+A} d \omega=I\left[\frac{\omega^{2}}{2}-\frac{A}{2} \ln \left(\omega^{2}+A\right)\right]_{0}^{\omega_{i}}$

where $A=K_{d} / K_{\mathrm{gw}}$. Since $E_{\mathrm{gw}}^{\mathrm{To}}$ amounts to a fraction $\alpha$ of the initial spin energy of the NS, we can write:

$(1-\alpha) \omega_{i}^{2}=A \ln \left(\frac{\omega_{i}^{2}+A}{A}\right)$

Finally, defining the ratio of the GW over magnetodipole torque as $x \equiv\left(\omega_{i}^{2} / A\right)$ :

$$
\begin{aligned}
x & \approx 2.25 \frac{B_{t, 16.3}^{4}}{B_{d, 14}^{2} P_{i, 2}^{2}} \\
1-\alpha & =\frac{\ln (1+x)}{x}
\end{aligned}
$$

The above expressions provide us with two relations between the five parameters $\left(\omega_{i}, B_{d}, B_{t}, x, \alpha\right)$. A third one derives from the fact that the remaining fraction $(1-\alpha)$ of the initial spin energy is available for being transferred to the ejecta through magnetodipole radiation. By assuming that all this energy is effectively transferred to the SNR, we get:

$\omega_{i}^{2}=\frac{2 E_{S N R}^{\mathrm{inj}}}{(1-\alpha) I}=\frac{2 x E_{S N R}^{\mathrm{inj}}}{\ln (1+x) I}$

where $E_{S N R}^{\mathrm{inj}}$ is the spin-down energy injected in the expanding shell, constrained by the results of Vink \& Kuiper
(2006) to be $\simeq 10^{51} \mathrm{erg}$. We use the results of Lattimer \& Prakash (2001), according to which a $1.4 \mathrm{M}_{\odot}$ NS has a radius $R \simeq$ $12 \mathrm{~km}$ and a moment of inertia $I \approx 0.35 M R^{2} \simeq 1.4 \times 10^{45}$ $\mathrm{g} \mathrm{cm}^{2}$. Note that, by these numbers, one obtains the following relation between the measured $P$ and $\dot{P}$ of NSs and the corresponding value of the dipolar magnetic field at the magnetic pole (our $\left.B_{d}\right)$ 1:

$B_{d} \simeq 4.4 \times 10^{19}(P \dot{P})^{\frac{1}{2}} \mathrm{G}$

As can be seen from Fig. (11), dipole magnetic fields stronger than $(5 \div 6) \times 10^{14} \mathrm{G}$ rapidly quench the expected $\mathrm{S} / \mathrm{N}$ ratio of GW signals from newly formed magnetars. Therefore, we restrict our investigation to polar dipole fields $B_{d}<6$. From Eq. (10) we see that, once $x$ is given, both $\alpha$ and $\omega_{i}$ are determined (one can indeed use any of the three as the free parameter). The first expression of Eq. (9) thus provides a relation between $B_{d}$ and $B_{t}$.

We have repeated this procedure for five values of the ratio of the GW over the magnetodipole torque $(x)$ and, for each of them, obtained the implied values of $\omega_{i}$ and $\alpha$ and a curve $B_{t}$ vs. $B_{d}$.

Fig. 2 summarizes our results. Loci in the $B_{d}$ vs. $B_{t}$ plane for which fast spinning, ultramagnetized magnetars are consistent with the energetic constraints derived

\footnotetext{
1 This is a factor 1.5 less than usually assumed with $I=$ $10^{45} \mathrm{~g} \mathrm{~cm}^{2}$ and $R=10 \mathrm{Km}$. Use of the most up to date parameters is required, given the strong dependence of the two competing torques on the exact value of the magnetic fields.
} 
by Vink \& Kuiper (2006) are drawn for the five chosen values of $x$ or, equivalently, the initial spin period (since $E_{S N}^{i n j}$ is fixed). Details are given in the caption.

In summary, we have first identified a range of initial conditions (spin period, internal and external magnetic field), within which newly formed magnetars can be interesting targets for next generation GW detectors. Then we have calculated that, within most of that same region, magnetars should emit less than $10^{51}$ ergs through magnetodipole radiation (cfr. Dall'Osso et al. (2007) and Fig. 21). This is compatible with the limits inferred through recent X-ray observations of SNRs around present-day magnetar candidates.

\section{Discussion}

The energy liberated in the 2004 December 27 flare from SGR 1806-20, together with the likely recurrence rate of these events, points to a magnetar internal field strength of $\sim 10^{16} \mathrm{G}$ or greater. Such a field likely results from differential rotation in a millisecond spinning proto-magnetar and deforms the star into a prolate shape. Magnetars with these characteristics are expected to be very powerful sources of gravitational radiation in the first days to weeks of their life. An evolving periodic GW signal at $\sim 1 \mathrm{kHz}$, whose frequency halves over weeks, would unambiguously reveal the early days of a fast spinning magnetar.

Prospects for revealing their GW signal depend on the birth rate of these objects. The three associations between an AXP and a supernova remnant (ages in the $10^{3} \div 10^{4}$ yr range) implies a magnetar birth rate of $\geq 0.5 \times 10^{-3} \mathrm{yr}^{-1}$ in the Galaxy (Gaensler et al. 1999). Therefore the chances of witnessing the formation of a magnetar in our Galaxy are slim. A rich cluster like Virgo, containing $\sim 2000$ galaxies, is expected to give birth to magnetars at a rate of $\geq 1 \mathrm{yr}^{-1}$. A fraction of these might have sufficiently high toroidal fields that a detectable GW is produced.

It has been recently found that SNR shells around some magnetar candidates have comparable expansion energies to standard SNR shells. This implies that either the NS was not initially spinning as fast as required for an $\alpha-\Omega$ dynamo to amplify its field to magnetar strengths, or that most of its initial spin energy was emitted in a way that did not interact with the ejecta. GWs have indeed such property. If the internal magnetic field of newly formed magnetars is $>10^{16} \mathrm{G}$, comparable to the lower limit estimated through the enegetics of the Dec 27 Giant Flare, then their GW emission can be strong enough to radiate away most of their initial spin energy. The required amount of energy emitted through GWs is indeed such that, had these magnetar candidates been at the distance of the Virgo cluster, they would have been revealed by a LIGO II-class GW detector.
Therefore, GWs from newly formed magnetars can account naturally for the recent X-ray observations of SNRs around galactic magnetars. The main conclusion that can be drawn at present is that newborn, fast spinning magnetars represent a potential class of GW emitters over Virgo scale distances that might well be within reach for the forthcoming generation of $\mathrm{GW}$ detectors.

\section{References}

Abbott,B. et al. [LIGO Scientific Collaboration] gr-qc/0508065 (2005)

Alpar, A., \& Pines, D. Nature, 314, 334 (1985)

Alpar, A., \& Sauls, J., A. Ap.J., 327, 723 (1988)

Arzoumanian, Z., Cordes, J.' M. \& Chernoff, D. F. Ap.J., 568, 289 (2002)

Bonazzola, S. \& Marck, J.A. Ann. Rev. Nucl. Part. Sci., 45, 655 (1994)

Bonazzola, S. \& Gourgoulhon, E. A.\&A., 312, 675 (1996)

Brady, P., Greighton, T., Cutler, C. \& Schutz, B.F. Phys. Rev. D, 57, 2101 (1998)

Brady, P.R. \& Creighton, T. Phys. Rev. D , 61, 082001 (2000)

Cutler, C. Phys. Rev. D, 66, 084025 (2002)

Cutler, C., Golami, I. \& Krishnan, B. gr-qc/0505082 (2005)

Dall'Osso, S., Re, V., submitted to Phys. Rev. D (2006)

Dall'Osso, S., et al., in preparation

Duncan, R., C. Ap.J.Letters, 498, L45 (1998)

Duncan,R.,C., \& Thompson,C. Ap.J.Letters, 392, L9 (1992)

Feroci, M., et al. Ap.J.Letters, 515, L9 (1999)

Ferrario, L.,\& Wickramasinghe, D. M.N.R.A.S., 367, 1323 (2006)

Gaensler, B., M., Gotthelf, E., V., \& Vasisht, G. Ap.J.Letters, 526, L37 (1999)

Hurley, K., et al. Nature, 397, , 41 (1999)

Hurley, K., et al. Nature, 434, 1098 (2005)

Jaranowski, P., Krolak, A., \& Schutz, B.F. Phys. Rev. D, 58, 063001 (1998)

Jones, D., I. 2002, Class.Quantum Grav., 19, 1255 (2002)

Jones, D. I., \& Andersson, N. M.N.R.A.S., 331, 203 (2002)

Konno, K., Obata, T., \& Kojima, Y. A.\&A., 356, 234 (2002)

Kouveliotou, C., et al. Nature, 393, 235 (1998)

Krishnan, B., Sintes, A.M., Papa, M.A., Schutz, B.F., Frasca, S., \& Palomba, C. Phys. Rev. D, 70082001 (2004)

Lattimer, J., M., Prakash, M., Ap.J., 500, 426 (2001)

Lyutikov, M. M.N.R.A.S., 346, 540 (2003)

Lazzati, D., Ghirlanda, G. \& Ghisellini, G. M.N.R.A.S., ,, in press (astro-ph/0504308 v2) (2005)

Mazets, E., P., Golentskii, S., V., Ilinskii, V., N., Aptekar, R., L., Guryan \& Iu., A. Nature, 282, 587 (1979)

Mereghetti, S., \& Stella, L. Ap.J.Letters, 442, L17 (1995)

Mestel, L., Takhar, H. S. M.N.R.A.S., 156, 419 (1972)

Nakar, E., Gal-Yam, A., Piran, T., Fox, D., B., astro-ph/0502148 v1 (2005)

Owen, B., J., \& Lindblom, L. Class.Quantum Grav., 191247 (2002)

Palomba, C., A.\&A., 367, 525 (2001)

Popov, S.B. \& Stern, B. E. M.N.R.A.S., in press (astro-ph/0503532 v3) (2005)

Stella, L., Dall Osso, S., Israel, G. L. \& Vecchio, A. Ap.J.Letters, 634, L165 (2005)

Tanvir, N. R., Chapman, R., Levan, A. J. \& Priddey, R. S. Nature, 438, 991 (2005)

Terasawa, T., et al. astro-ph/0502315 (2005)

Thompson, C., \& Duncan, R.,C. Ap.J., 408, 194 (1993) 
Thompson, C., \& Duncan, R. C. M.N.R.A.S., 275, 255 (1995)

Thompson, C., \& Duncan, R. C. Ap.J., 473, 322 (1996)

Thompson, C., \& Duncan, R. C. Ap.J., 561, 980 (2001)

Thompson, C., \& Murray, N. C. Ap.J., 560, 339 (2001)

Terasawa, T., et al. Nature, 434, 1110 astro-ph/0502315 (2005)

Thompson, T., A., Chang, P., Quataert, E. Ap.J., 611, 380 (2004)

Thorne, K.S. LIGO Internal Document G000025-00-M (2000)

Ushomirsky, G., Cutler, C., \& Bildsten, L. M.N.R.A.S., 319, 902 (2000)

Vink, J., \& Kuiper, L. M.N.R.A.S., 370, L14 (2006)

Woods, P., M., \& Thompson, C. (astro-ph/0406133 v3) (2004) 